\title{
Structural phase transition in monolayer gold(I) telluride: From a room-temperature topological insulator to an auxetic semiconductor
}

\author{
Xin Chen $\odot,{ }^{1}$ Raquel Esteban-Puyuelo $\odot,{ }^{1}$ Linyang Li $\odot, 2,{ }^{2}$ and Biplab Sanyal $\odot^{1, \dagger}$ \\ ${ }^{1}$ Department of Physics and Astronomy, Uppsala University, Box 516, 75120 Uppsala, Sweden \\ ${ }^{2}$ School of Science, Hebei University of Technology, Tianjin 300401, China
}

(Received 7 September 2020; revised 8 December 2020; accepted 5 January 2021; published 19 February 2021)

\begin{abstract}
Structural phase transitions between semiconductors and topological insulators have rich applications in nanoelectronics but are rarely found in two-dimensional (2D) materials. In this work, by combining ab initio computations and evolutionary structure search, we investigate two stable $2 \mathrm{D}$ forms of gold(I) telluride $\left(\mathrm{Au}_{2} \mathrm{Te}\right)$ with square symmetry, noted as $s(\mathrm{I})$ - and $s(\mathrm{II})-\mathrm{Au}_{2} \mathrm{Te} . \mathrm{s}(\mathrm{II})-\mathrm{Au}_{2} \mathrm{Te}$ is the global minimum structure and is a room-temperature topological insulator. $\mathrm{s}(\mathrm{I})-\mathrm{Au}_{2} \mathrm{Te}$ is a direct-gap semiconductor with high carrier mobilities and unusual in-plane negative Poisson's ratio. Both s(I) and s(II) phases have ultralow Young's modulus, implying high flexibility. By applying a small tensile strain, $\mathrm{s}(\mathrm{II})-\mathrm{Au}_{2} \mathrm{Te}$ can be transformed into $\mathrm{s}(\mathrm{I})-\mathrm{Au}_{2} \mathrm{Te}$. Hence, a structural phase transition from a room-temperature topological insulator to an auxetic semiconductor is found in the 2D forms of $\mathrm{Au}_{2} \mathrm{Te}$, which enables potential applications in phase-change electronic devices. Moreover, we elucidate the mechanism of the phase transition with the help of phonon spectra and group theory analysis.
\end{abstract}

DOI: 10.1103/PhysRevB.103.075429

\section{INTRODUCTION}

Graphene has been proved or predicted to have a lot of exciting properties since its first realization, such as large Young's modulus, high mobility, and quantum spin Hall (QSH) effect [1-4], to name a few. The QSH effect is observed in two-dimensional topological insulators (2DTIs), which are a new state of matter with time-reversal symmetry protected edge states [4-7]. Though the QSH effect was first predicted in graphene by Kane and Mele [4], it has not been observed in experiments due to the ultraweak spin-orbit coupling (SOC) effect in carbon systems. In subsequent works, the QSH effect was observed in $\mathrm{HgTe} / \mathrm{CdTe}$ [8] and InAs/GaSb [9] quantum wells at ultralow temperatures $(1.4 \mathrm{~K})$. Very recently, the QSH effect has been achieved in monolayer $\mathrm{WTe}_{2}$ [10], at about $100 \mathrm{~K}$, which is a significant improvement but still much lower than the room temperature. Till now, many other materials have been theoretically predicted to be room-temperature 2DTIs [11-15]. Most of the predictions have not been verified by experiments yet, but we can expect them to be realized in the future.

Phase transition materials (PTMs) are the materials exhibiting several stable crystalline phases with distinct properties and similar total energy. PTMs have attracted elated

\footnotetext{
*linyang.li@ hebut.edu.cn

†biplab.sanyal@physics.uu.se
}

Published by the American Physical Society under the terms of the Creative Commons Attribution 4.0 International license. Further distribution of this work must maintain attribution to the author(s) and the published article's title, journal citation, and DOI. Funded by Bibsam. research interest due to the promise for the applications in electronic and optical devices and sensors [11,16-19]. Among the reported PTMs, the 3D metal-insulator PTMs $\mathrm{VO}_{2}$ and $\mathrm{TaS}_{2}$ have been used as the channel materials for fabricating phase transition devices [20,21]. The 2D metal-insulator phase transition in $\mathrm{MoTe}_{2}$ is also realized by thermal means [22,23], electrostatic doping [16], electrostatic gating [19,24,25], and photoexcitation [26]. Moreover, semiconductor-TI PTMs, as a new group of materials, have attracted much attention since they are an excellent platform to manipulate the topological properties of $2 \mathrm{D}$ materials and realize Majorana bound modes [26-28]. However, as a new class of materials, 2DTIs have rarely been reported as a stable phase of PTMs in experimental or theoretical works [24,29 31].

$M_{2} X(M=$ metal, $X=$ nonmetal $)$ monolayers are a new class of 2D materials that have been given a lot of attention $[32,33]$. The 2D forms of group IB-VIA compounds have numerous $M_{2} X$ phases. $2 \mathrm{D} \beta-\mathrm{Cu}_{2} \mathrm{~S}$ and $\gamma-\mathrm{Cu}_{2} \mathrm{~S}$ sheets have been synthesized by Romdhane et al. and Li et al. [34,35]; $\alpha-\mathrm{Ag}_{2} \mathrm{~S}$ sheets have been synthesized by Feng et al. via the liquid-phase exfoliation method [36]. Zhu et al. synthesized 2D colloidal $\mathrm{Cu}_{2} \mathrm{Se}$ using the Langmuir-Blodgett (LB) method [37]. 2D Cu $2 \mathrm{Te}$ was synthesized by Qian et al., using the molecular beam epitaxy method [38]. Besides the experimental studies, theoretical works based on density functional theory and global structure search $[39,40]$ have predicted several monolayer $M_{2} X$ with lower energy than the experimentally synthesized materials [41-44]. In a recent article, the $2 \mathrm{D}$ forms of $M_{2} X(X=\mathrm{Cu}, \mathrm{Ag}, \mathrm{Au} ; X=\mathrm{S}, \mathrm{Se})$ materials have been discussed [42]. It is found that there are two kinds of structures with square symmetry, i.e., the $P 4 / \mathrm{nmm}$ group structure s(I) and $P 4212$ group structure s(II). For copper(I) sulfide and selenide, $\mathrm{s}(\mathrm{II})$ structures are energetically favored, 
while for silver(I) and gold(I) compounds, s(I) structures are favored. Due to the absence of an energy barrier between $\mathrm{s}$ (I) and s(II) phases in most of these materials, the system will always go to one of the phases. As an exception, s(II)$\mathrm{Au}_{2} \mathrm{Se}$ is dynamically stable, but s(I) and s(II) phases are both direct-gap semiconductors, which makes them not suitable as a semiconductor-TI PTM. Nevertheless, this inspires us to investigate other $M_{2} X$ materials with stronger relativistic effects.

In this work, based on global structure search using an evolutionary algorithm $[45,46]$, we have predicted two stable phases of $\mathrm{Au}_{2} \mathrm{Te}, \mathrm{s}(\mathrm{I})$ and $\mathrm{s}(\mathrm{II})$. The energy difference between these two phases is as low as $4 \mathrm{meV} /$ atom. We have proposed a structural phase transition from $s(\mathrm{II})-$ to $\mathrm{s}(\mathrm{I})-\mathrm{Au}_{2} \mathrm{Te}$ by external tensile strain and a reverse phase transition by chemical means at low temperature. We further investigated the influence of electric field in the phase transition, and it is found that with the application of electric field larger than $0.7 \mathrm{~V} / \AA$, s(I) has lower energy than $\mathrm{s}(\mathrm{II})$, which makes the $s(\mathrm{II}) \rightarrow \mathrm{s}(\mathrm{I})$ phase transition possible with the assistance of heat. Moreover, it is found that they are quite different in their electronic and mechanical properties. $\mathrm{s}(\mathrm{I})-\mathrm{Au}_{2} \mathrm{Te}$ is a direct-gap semiconductor with high electron and hole mobilities of $3.45 \times 10^{4} \mathrm{~cm}^{2} /(\mathrm{V} \mathrm{s})$ and $6.47 \times 10^{3} \mathrm{~cm}^{2} /(\mathrm{V} \mathrm{s})$, while the $\mathrm{s}(\mathrm{II})$ phase is a TI with a large nontrivial band gap of $28.4 \mathrm{meV}$. Both of the two structures have an ultralow Young's modulus, showing extremely high flexibility. But by applying strain, only the s(I) phase shows an unusual negative Poisson's ratio (NPR). Finally, we computed the Raman spectra of them and have shown that the peak at about $65.9 \mathrm{~cm}^{-1}$ is only observed in the s(II) phase, which can be an excellent method to distinguish the two similar phases.

\section{COMPUTATIONAL DETAILS}

We performed the global structure search with the evolutionary algorithm based code USPEX [45-49] to obtain low-energy stable structures. In the structure search, the unit cell is chosen to contain four $\mathrm{Au}$ atoms and two Te atoms, and the initial thickness of the region is set as $0-4 \AA$. The population size is set to 30 . The structure search is converged if the ground state structure did not change for 10 generations. All the first-principles calculations are performed by using the density functional code VASP [50,51]. The generalized gradient approximation in the form of Perdew, Burke, and Ernzerhof (PBE) [52] is used for the exchange-correlation potential. For the calculations of $2 \mathrm{D}$ materials, the out-ofplane interaction is avoided by taking a vacuum of more than $20 \AA$. The energy cutoff of the plane waves is set to more than $450 \mathrm{eV}$. The tolerance for energy convergence is set to be less than $10^{-5} \mathrm{eV}$. We optimized the structures until the force on each atom becomes smaller than $0.001 \mathrm{eV} / \AA$. The Brillouin zone (BZ) is sampled by using the Monkhorst-Pack grid denser than $2 \pi \times 0.033 \AA^{-1}$. For examining the dynamical stability of structures, phonon spectra are computed using the PHONOPY code [53]. In the calculations of the electronic band structures, the Heyd-Scuseria-Ernzerhof (HSE) hybrid functional within the framework of HSE06 [54] is employed. Postprocessing of some calculations is performed by using VASPKIT [55] and VESTA [56].
TABLE I. The lattice parameters ( $a_{x}$ and $a_{d}$, in $\AA$ ), slab thicknesses $(h$, in $\AA$ ), distortion angles $(\theta$, in deg), cohesive energies $(\Delta E$, in $\mathrm{eV} /$ atom), and stiffness tensors $(\mathrm{C} 11, \mathrm{C} 12$, and $\mathrm{C} 66$, in $\mathrm{N} / \mathrm{m})$ of $\mathrm{s}(\mathrm{I} / \mathrm{II})-\mathrm{Au}_{2} \mathrm{Te}$ monolayers.

\begin{tabular}{lcccccccc}
\hline \hline Phase & $a_{x}$ & $a_{d}$ & $h$ & $\theta$ & $\Delta E$ & $\mathrm{C} 11$ & $\mathrm{C} 12$ & $\mathrm{C} 66$ \\
\hline $\mathrm{s}(\mathrm{I})$ & 5.85 & 8.27 & 3.44 & 0 & -2.893 & 23.460 & 5.953 & 12.301 \\
$\mathrm{~s}(\mathrm{II})$ & 5.61 & 7.93 & 3.55 & 15.28 & -2.897 & 34.798 & 19.006 & 14.199 \\
\hline \hline
\end{tabular}

\section{RESULTS AND DISCUSSION}

\section{A. Structure and stability}

Among the hundreds of structures generated in the evolutionary structural search for $\mathrm{Au}_{2} \mathrm{Te}$, the square symmetry structures s(I) and s(II), as shown in Fig. 1(a), are found to be energetically favored, as shown in Fig. S1 in the Supplemental Material (SM) [57]. s(I)- and s(II)- $\mathrm{Au}_{2} \mathrm{Te}$ are the secondlowest and the lowest energy structures, showing $P 4 / \mathrm{nmm}$ and $P 4212$ space group symmetry, respectively. In detail, there are four $\mathrm{Au}$ atoms sandwiched by two Te atoms in one s(I/II)$\mathrm{Au}_{2} \mathrm{Te}$ unit cell. The relationship between s(I) and s(II) phases is like that between the $1 T$ and $1 T^{\prime}$ phases of $\mathrm{MoTe}_{2}$; i.e., the symmetry of the structures is reduced by distortion. The critical parameters of the two structures are listed in Table I. The lattice constant of the $\mathrm{s}(\mathrm{I})$ phase is $4.3 \%$ larger than that of s(II), while the thickness of the former is $3.1 \%$ smaller than the latter. The electron localization functions (ELFs) are shown in Fig. 1(b), where it is observed that the electrons are mainly localized near Te atoms, but the electron density near $\mathrm{Au}$ is much more than that near the $\mathrm{Cu}$ and $\mathrm{Ag}$ atoms in s(II)$\mathrm{Cu}_{2} \mathrm{~S}$ and $\mathrm{s}(\mathrm{I})-\mathrm{Ag}_{2} \mathrm{~S}$ monolayers. This can be explained by the much lower electronegativity of tellurium atoms compared to that of sulfur atoms.

To study the energetic stability and compare to other $2 \mathrm{D}$ materials, we compute the cohesive energy following the expression

$$
\Delta E=\frac{E_{\mathrm{Au}_{2} \mathrm{Te}}-\left(n_{\mathrm{Au}} \times E_{\mathrm{Au}}+n_{\mathrm{Te}} \times E_{\mathrm{Te}}\right)}{n_{\mathrm{Au}}+n_{\mathrm{Te}}} .
$$

In this formula, $E_{\mathrm{Au}_{2} \mathrm{Te}}$ is the total energy of $\mathrm{s}(\mathrm{I} / \mathrm{II})-\mathrm{Au}_{2} \mathrm{Te}$; $E_{\mathrm{Au}}$ and $E_{\mathrm{Te}}$ are the energies of the $\mathrm{Au}$ and Te atoms. The values of $\Delta E$ are listed in Table I. For comparison, the values of the synthesized germanene and stanene are computed as $-3.26 \mathrm{eV} /$ atom and $-2.74 \mathrm{eV} /$ atom, respectively [58]. The low cohesive energy implies that the synthesis of $s(\mathrm{I} / \mathrm{II})$ $\mathrm{Au}_{2} \mathrm{Te}$ is highly probable.

To further strengthen the aspect of the energetic stability of $\mathrm{s}(\mathrm{I} / \mathrm{II})-\mathrm{Au}_{2} \mathrm{Te}$, we performed a variable-composition structure search with a unit cell containing up to $6 \mathrm{Au}$ or Te atoms. The objective function was defined as $E_{f}=\left[E\left(\mathrm{Au}_{x} \mathrm{Te}_{y}\right)-\right.$ $x E(\mathrm{Au})-y E(\mathrm{Te})] /(x+y)$, in which $E(\mathrm{Au})$ and $E(\mathrm{Te})$ are the total energies of the ground state structures of $2 \mathrm{D} \mathrm{Au}$ and Te structures, respectively. From the convex hull diagram, as shown in Fig. S5 of the SM, it is confirmed that s(II)$\mathrm{Au}_{2} \mathrm{Te}$ is one of the most stable structures in the 2D Au-Te system.

The dynamic stability of $\mathrm{s}(\mathrm{I} / \mathrm{II})-\mathrm{Au}_{2} \mathrm{Te}$ is confirmed by the density functional perturbation theory (DFPT) calculations and $a b$ initio Born-Oppenheimer molecular dynamics 


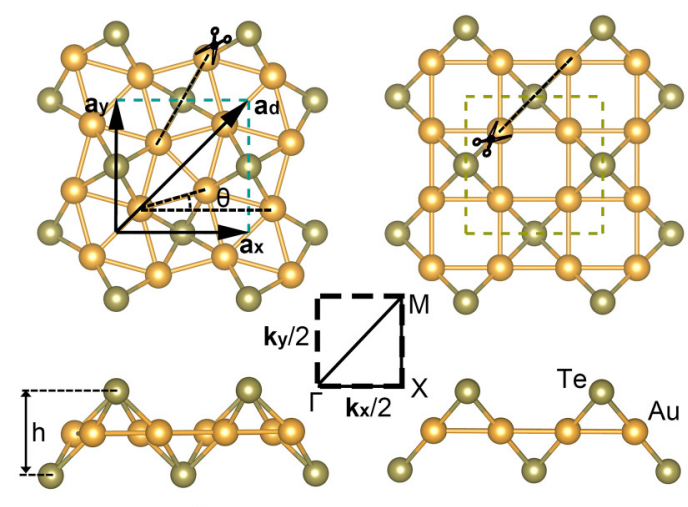

(a)

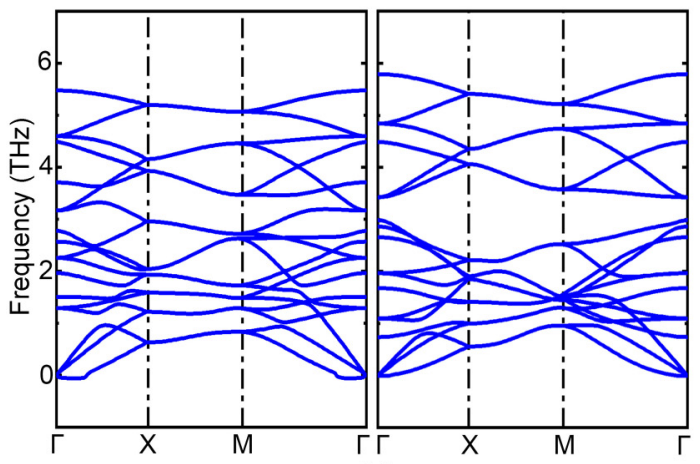

(c)

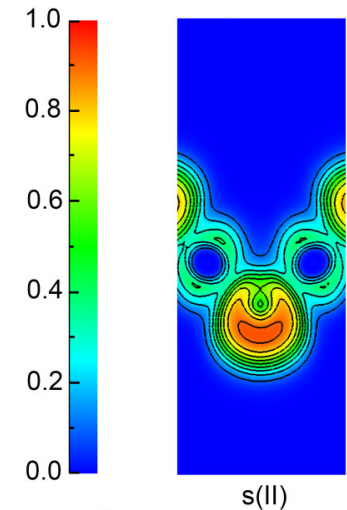

(b)

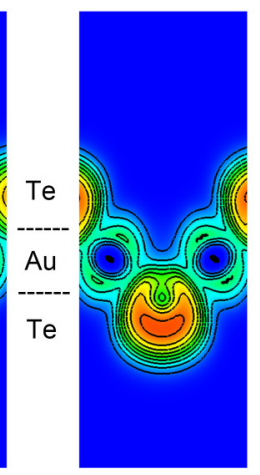

$s(I)$

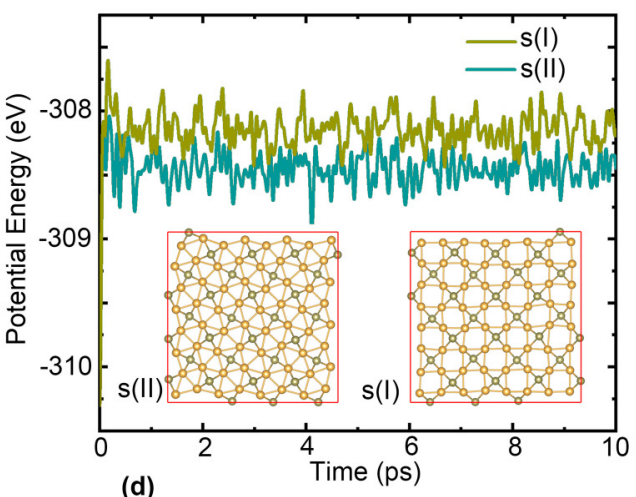

(d)

FIG. 1. (a) The top and side views of the atomic structures of $\mathrm{s}(\mathrm{II})-$ and $\mathrm{s}(\mathrm{I})-\mathrm{Au}_{2} \mathrm{Te}$ monolayers with their primitive cell marked by dashed square. $\mathbf{a}_{\mathbf{x}}, \mathbf{a}_{\mathbf{y}}, \mathbf{a}_{\mathbf{d}}, h$, and $\theta$ are the lattice vectors in the $x, y$, and diagonal directions, the heights, and the distortion angles of s(I/II)-Au $\mathrm{A}_{2} \mathrm{Te}$. The middle dashed black square is the first $\mathrm{BZ}$ of $\mathrm{s}(\mathrm{I} / \mathrm{II})-\mathrm{Au}_{2} \mathrm{Te}$ monolayers, with the high-symmetry points indicated. (b) The contour maps of ELFs of $s(I I)-$ and $s(\mathrm{I})-\mathrm{Au}_{2} \mathrm{Te}$, which are sliced on $\mathrm{Au}-\mathrm{Te}-\mathrm{Au}$ planes marked with scissors in (a). (c) The phonon dispersion of $\mathrm{s}(\mathrm{I} / \mathrm{II})-\mathrm{Au} \mathrm{u}_{2} \mathrm{Te}$. (d) The potential energy change as a function of simulation time at $300 \mathrm{~K}$ in BOMD simulations along with the final structures.

(BOMD) simulations. In the DFPT calculations, we employed a $3 \times 3 \times 1$ supercell and a $3 \times 3 \times 1 \Gamma$-centered $k$ mesh. As shown in Fig. 1(c), in the phonon spectra of both s(I) and $\mathrm{s}$ (II) phases, there are no or only tiny imaginary frequencies (less than $0.1 \mathrm{THz}$ ) near the $\Gamma$ point. In the BOMD simulations, we have checked the structural and energetic change of the $4 \times 4 \times 1$ supercells of s(I)- and s(II)- $\mathrm{Au}_{2} \mathrm{Te}$ at $300 \mathrm{~K}$ until $10 \mathrm{ps}$, with a time step of $1 \mathrm{fs}$. As shown in Fig. 1(d), the energies of the two phases are kept stable, and there is no obvious structural change in the final geometrical framework.

A mechanically stable 2D material should satisfy the BornHuang criteria: the elastic modulus tensor components $C_{11}$, $C_{22}$, and $C_{66}$ should be positive, and $\left|C_{11}+C_{22}\right|>\left|2 C_{12}\right|$. We fitted the curves of the energy changes $U$ versus strains $\tau_{x y}$ using the formula $[59,60]$

$$
U=\frac{1}{2} C_{11} \tau_{x}^{2}+\frac{1}{2} C_{22} \tau_{y}^{2}+C_{12} \tau_{x} \tau_{y}+2 C_{66} \tau_{x y}^{2} .
$$

Considering the square symmetry, $C_{22}$ is equal to $C_{11}$, and the rest of the components of stiffness tensors are listed in Table I. The results meet the criteria, confirming that both phases of $\mathrm{Au}_{2} \mathrm{Te}$ are mechanically stable. In the light of other 2D compounds of transition metal and group IVA elements, we suggest that the chemical vapor deposition method could be a possible way to synthesize s(I/II)- $\mathrm{Au}_{2} \mathrm{Te}[34,61,62]$.

\section{B. Structural phase transition}

Notably, the energy difference between the two phases is just $4 \mathrm{meV}$. With similar structures and energies, the phase transition between the two phases is quite promising. Considering the obvious difference between the lattice parameters of the two phases, we investigated the feasibility of introducing the phase transition from s(II) to s(I) structure by changing the lattice parameters (applying external tensile strain), and the energy change in the process is shown in Fig. 2(a). Interestingly, it is found that by applying a tensile strain of above about $6.5 \%$ along the (110) direction, the s(II) structure can be transformed into the $\mathrm{s}(\mathrm{I})$ structure, with an energy barrier of about $19 \mathrm{meV} /$ atom.

The s(I) phase is a more symmetric phase, belonging to the $D_{4 h}$ point group. In comparison, the s(II) phase can be classified as the $D_{4}$ point group, which is a subgroup of $D_{4 h}$. The difference between the two point groups is that the $D_{4}$ is not invariant under inversion symmetry, mirror plane symmetries, and therefore improper symmetries (which are a combination of a mirror plane symmetry and a rotation), whereas $D_{4 h}$ has all these symmetries. To connect the symmetry arguments with eigenmodes of phonons, we further computed the phonon spectra of $\mathrm{s}(\mathrm{II})-\mathrm{Au}_{2} \mathrm{Te}$ with different diagonal strains $\left(\epsilon_{d}\right)$ to investigate the phonon softening in the strain-induced phase transition process, as shown in Fig. 3 and Fig. S6 in the 

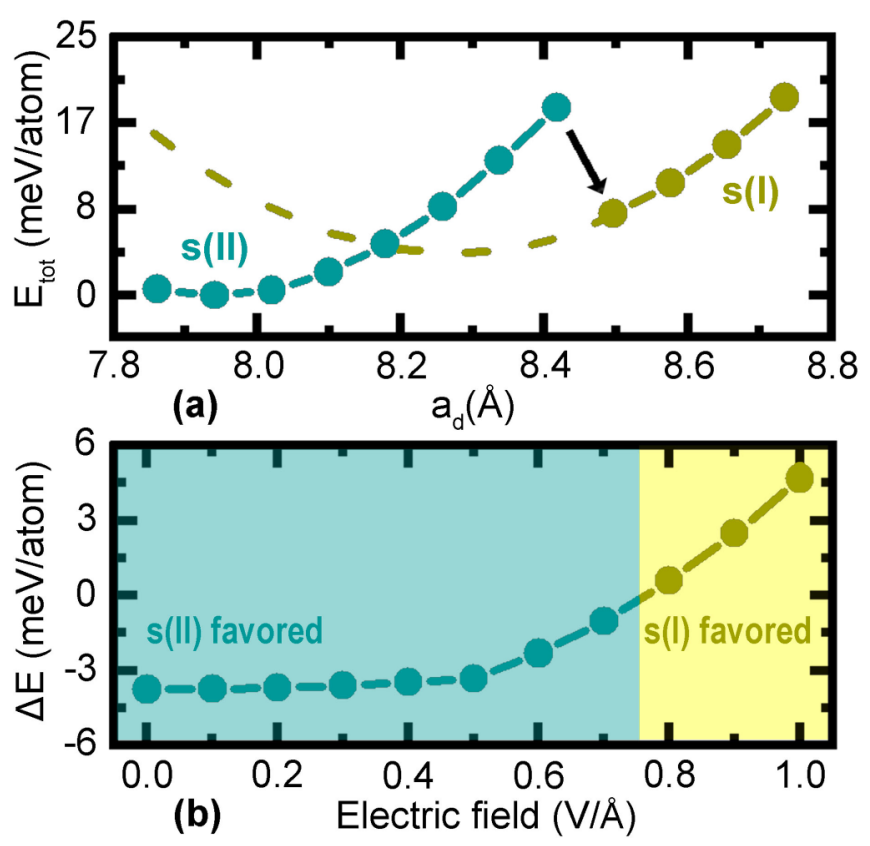

FIG. 2. (a) The energy change of the structure as a function of diagonal lattice constant $a_{d}$. The two local minima represent the fully relaxed $s(\mathrm{I})-$ and $s(\mathrm{II})-\mathrm{Au}_{2} \mathrm{Te}$ structures. (b) The energy difference between the two phases as a function of the strength of the electric field in the $z$ direction.

SM [57]. The diagonal strain lowers the symmetry from $D_{4}$ to $D_{2}$, where even fewer rotations leave the structure invariant. It is observed that with a strain of $6 \%, \mathrm{~s}(\mathrm{II})-\mathrm{Au}_{2} \mathrm{Te}$ is still
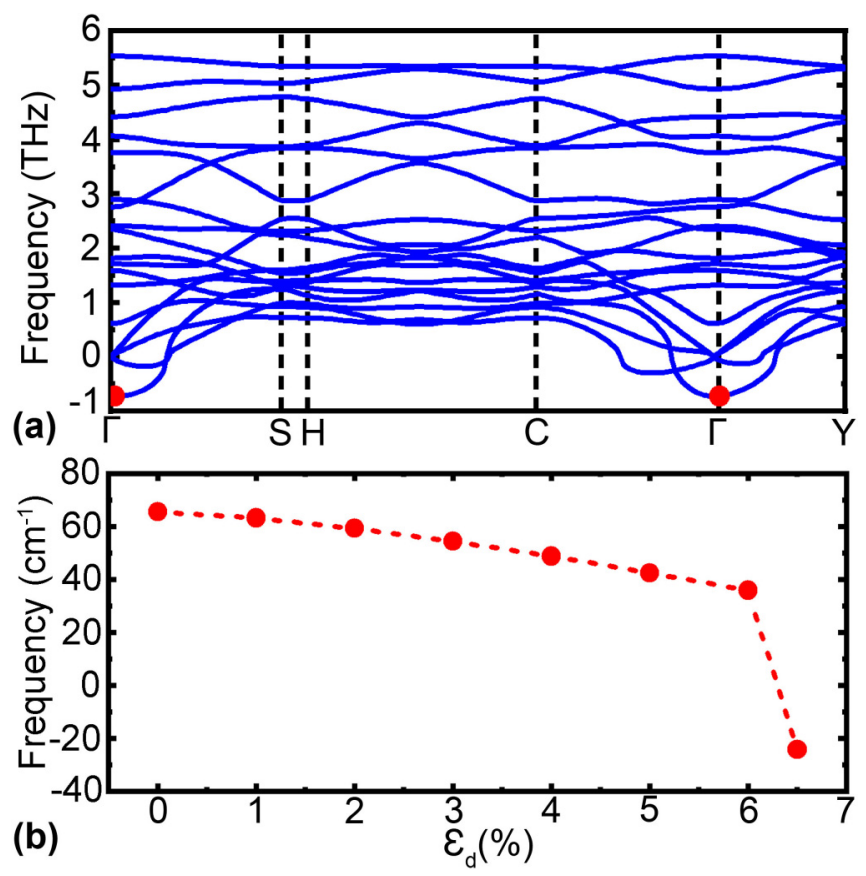

FIG. 3. (a) The phonon spectrum of $s(\mathrm{II})-\mathrm{Au}_{2} \mathrm{Te}$ with diagonal strain $\epsilon_{d}$ of $6.5 \%$ along the high-symmetry path as shown in Fig. S6(c). The red dots mark the phonon softening, and the corresponding phonon eigenvector is shown in the movie file "epsilon6.5.mp4" in the SM [57]. (b) The frequency of the phonon mode of the phasetransition eigenvector as a function of diagonal strain. stable. With a strain of $6.5 \%$, there is a softened phonon mode near the $\Gamma$ point. The eigenvector of the mode at the $\Gamma$ point is shown in the attached movie file "epsilon6.5.mp4" in the SM [57], which is actually the phase transition displacement from s(II) to s(I). By tracking the frequencies of this phonon mode with the special eigenvector under varying diagonal strains, as shown in Fig. 3(b), we have observed that the strain lowers the energy of this branch continuously until it reaches $6.5 \%$, where the abrupt transition happens. Therefore, even if the phonon mode allows the transition to be present at all stages, it is not until it is lowered enough in energy that the system undergoes the distortion that promotes the change of phase. With a strain of $7 \%$, the structure is transferred into the $\mathrm{s}(\mathrm{I})$ phase, and no imaginary frequencies are observed.

Compared to tensile strain, applying external compressive strain is much harder $[63,64]$. Therefore, to achieve the phase transition from the $\mathrm{s}(\mathrm{I})$ to $\mathrm{s}(\mathrm{II})$ structure, heating the system seems to be more achievable than applying compressive strain. By using the variable-cell nudged elastic band method (VC-NEB) [65], we investigated the phase transition mechanism between $s(\mathrm{I})$ and $\mathrm{s}(\mathrm{II})$. As shown in Fig. S2 in the Supplemental Material [57], the energy barrier of the transition from $\mathrm{s}(\mathrm{I})$ to $\mathrm{s}(\mathrm{II})$ is about $7.8 \mathrm{meV} /$ atom. Since the elastic constants of $s(\mathrm{I})$ are softer than that of $\mathrm{s}(\mathrm{II})$, the free energy of s(I) decreases faster than that of s(II). Based on the quasiharmonic approximation, we investigated the influence of temperature on the Helmholtz free energies, as shown in Fig. 4(a). It is found that the free energy of the s(I) phase decreases faster than the $\mathrm{s}(\mathrm{II})$ phase, which makes the $\mathrm{s}(\mathrm{I})$ phase more stable (about $4 \mathrm{meV} /$ atom lower) than the s(II) phase at $300 \mathrm{~K}$. Thus we cannot state that a s(I)-s(II) phase transition can be achieved by thermal excitation only. Instead, decreasing the temperature and with the help of some chemical methods such as catalysis, we may achieve the phase transition from s(II) to s(I), as shown in Fig. 4(b).

Notably, the higher total energy of the s(II) phase does not mean that the $s($ II) phase will transfer to the $s(\mathrm{I})$ phase at room temperature since the energy barrier is much larger than the total energy difference (4 meV/atom) at $300 \mathrm{~K}$. We can see the evidence in the BOMD simulation, even when the lattice parameters are fixed. The lattice parameter of the s(I) phase is $4.3 \%$ larger than the $s(\mathrm{I})$ phase; thus if the $\mathrm{s}(\mathrm{II})$ phase were not thermally stable, the $2 \mathrm{D}$ structure would buckle and wrinkle, which is not observed in the final structure. Moreover, we have performed an isobaric-isothermic BOMD simulation with variable lattice parameters $[66,67]$. It is found that during the simulation time up to $10 \mathrm{ps}$, the s(II) phase is always stable, and the phase transition from s(II) to s(I) is not observed. The relevant details are shown in Fig. S7 in the SM [57].

Moreover, the phase transition driven by the electric field has been given extensive attention due to the excellent connection with the electronic industry [19,24,25]. In this work, by applying the external electric field in the $z$ direction, we studied the energetic properties of $s(\mathrm{I})-$ and $s(\mathrm{II})-\mathrm{Au}_{2} \mathrm{Te}$. The energy change and the energy difference between the $\mathrm{s}(\mathrm{I})$ and $\mathrm{s}$ (II) phases are shown in Fig. 2(b). It is found that the energies of the two phases have similar energy-field curves, but the energy of the s(I) phase decreases sharper than that of the s(II) 

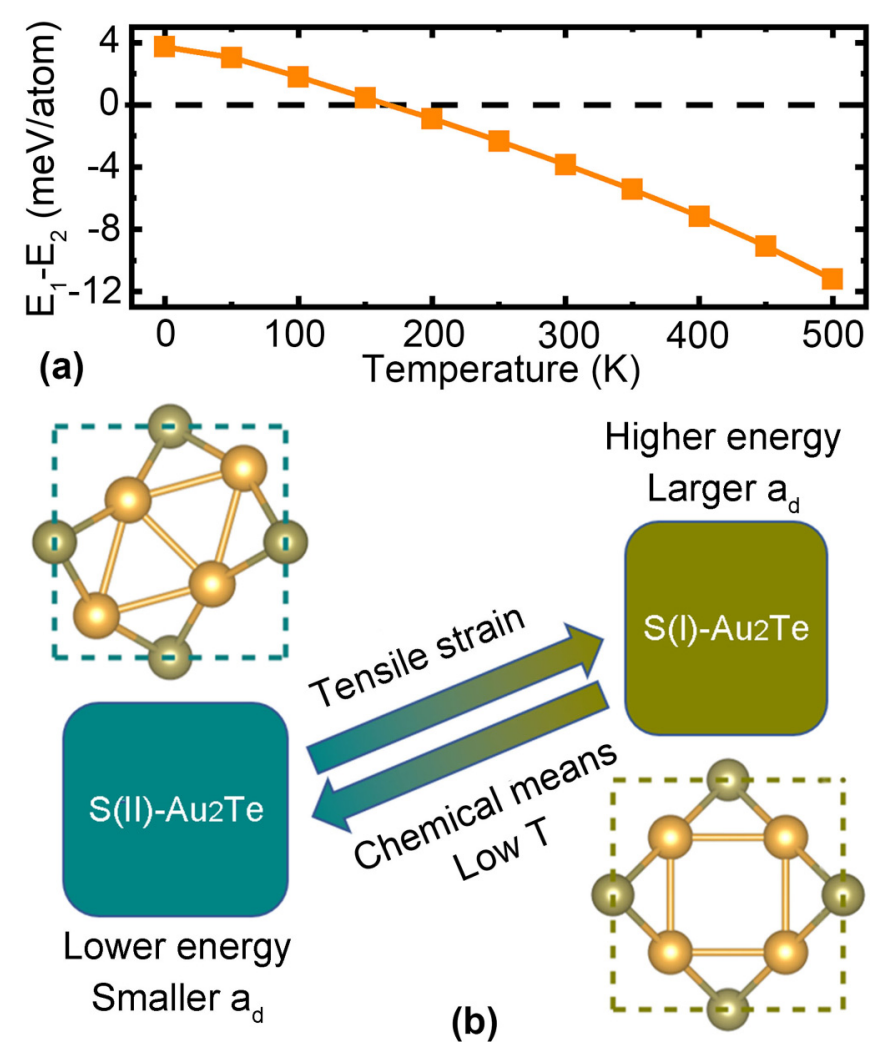

FIG. 4. (a) The free energy difference between $s(\mathrm{I})$ and $s($ II) as a function of temperature. (b) The mechanism of the reversible structural phase transition is indicated by mechanical and thermal means.

phase, which makes s(I) more energetically favored. Thus with the help of the external electric field, the $s($ II $) \rightarrow s(I)$ phase transition can be easier. However, achieving an ultrastrong electric field is very hard in the experiment, which means that in the $2 \mathrm{D} \mathrm{Au} \mathrm{Au}_{2} \mathrm{Te}$ case, the electric field is just an assistant method to make the phase transition easier, and it cannot drive the phase transition alone.

\section{Electronic and topological properties}

The electronic band structures of $2 \mathrm{D} s(\mathrm{I} / \mathrm{II})-\mathrm{Au}_{2} \mathrm{Te}$ are computed using the HSE06 method with and without the SOC effect taken into account, as shown in Fig. 5. s(I)-Au $\mathrm{Au}_{2}$ is a direct-gap semiconductor, with the valence band maximum (VBM) and conduction band minimum (CBM) both at the Gamma point, similarly to other reported $\mathrm{s}-A_{2} B$ structures $[34,35,41,43]$. The relativistic effect is prominently seen in the band structures due to the large atomic mass. Without SOC, the band gap is as large as $1.470 \mathrm{eV}$; with SOC, the band gap is decreased to $1.252 \mathrm{eV}$.

The electronic conductivity is a crucial property for the application of semiconductors. We computed the carrier mobilities by using the deformation potential method [68,69]:

$$
\mu=\frac{e \hbar^{3} C_{11}}{k_{B} \mathrm{~T} m^{*} m_{d}^{*}\left(E_{1}^{n}\right)^{2}},
$$

where $C_{11}$ is the $2 \mathrm{D}$ elastic modulus given in Eq. (1). $e, \hbar$, $k_{B}$, and $T$ are the electron charge, reduced Plank constant, Boltzmann constant, and temperature $(300 \mathrm{~K})$, respectively. $m^{*}$ and $m_{d}^{*}$ are the effective mass the average effective mass derived from $m_{d}^{*}=\sqrt{m^{*} m_{\perp}^{*}}$, where $m_{\perp}^{*}$ is the effective mass in the perpendicular direction of the transport direction. $E_{1}^{n}$ is the deformation potential constant of band $n$, and is defined by $E_{1}^{n}=\Delta V^{n} /\left(\Delta l / l_{0}\right)$, where $\Delta V^{n}$ is the change of the edge of band $n$ under deformation $\Delta l$. The transport direction is assumed as the $x$ direction. The electronic transport properties are determined by the computed quantities as $m_{d, e}^{*}=m_{e}^{*}=0.082 m_{0}, E_{1, e}=1.433 \mathrm{eV}$, and thus $\mu_{e}=3.45 \times 10^{4} \mathrm{~cm}^{2} /(\mathrm{V} \mathrm{s})$, whereas for the hole transport, the corresponding quantities are computed as $m_{d, h}^{*}=$ $m_{h}^{*}=-0.146 m_{0}, E_{1, h}=-2.029 \mathrm{eV}$, and thus $\mu_{h}=6.466 \times$ $10^{3} \mathrm{~cm}^{2} /(\mathrm{V} \mathrm{s})$. The mobilities are much larger than that of $1 \mathrm{H}-\mathrm{MoS}_{2}$ and black phosphorus [70-72], promising potential applications in nanodevices and new generation solar cells.

On the other hand, the electronic band structures of $\mathrm{s}(\mathrm{II})-\mathrm{Au}_{2} \mathrm{Te}$ calculated without and with SOC are shown in Fig. 5(b). Without SOC, both VBM and CBM are at the

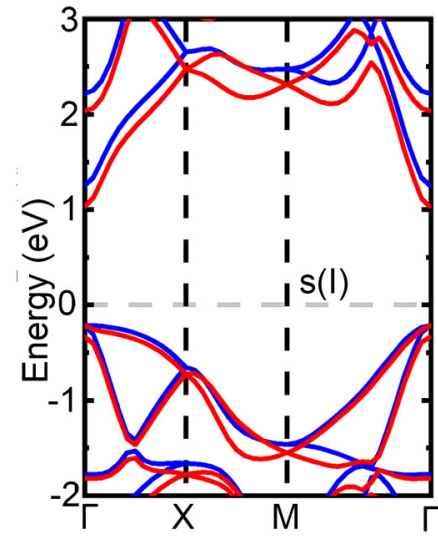

(a)

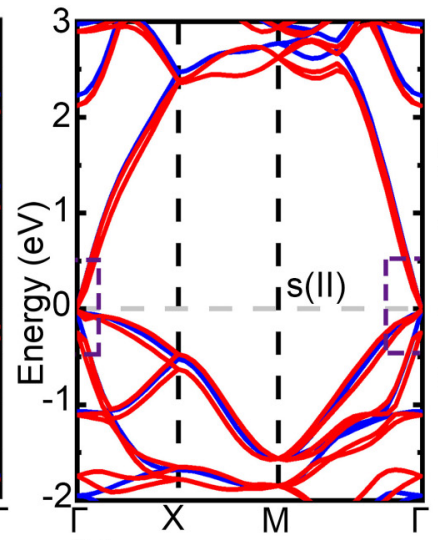

(b)

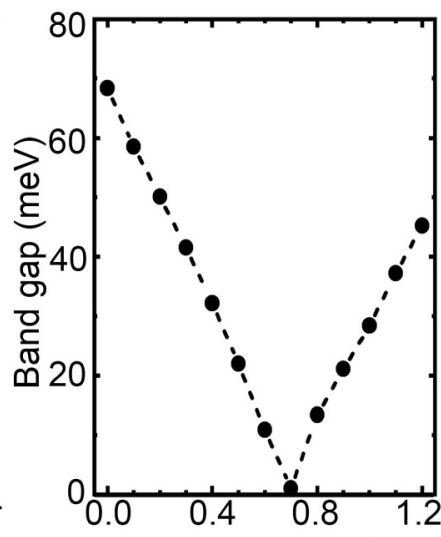

(c) SOC strength

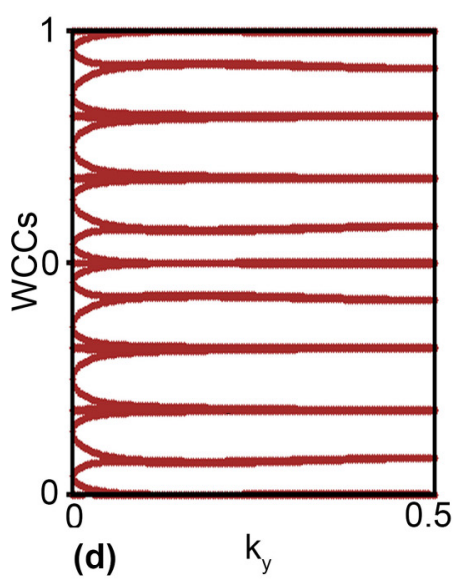

(d)

FIG. 5. The electronic band structures of (a) $s(\mathrm{I})-\mathrm{Au}_{2} \mathrm{Te}$ and (b) $\mathrm{s}(\mathrm{II})-\mathrm{Au}_{2} \mathrm{Te}$. The purple rectangle parts of $\mathrm{s}(\mathrm{II})-\mathrm{Au}_{2} \mathrm{Te}$ band structure are enlarged and shown in Fig. S4 in the SM [57]. The contribution of each atomic orbital is shown by the local density of states (LDOS) in Fig. S2 in the SM [57]. The blue and red lines are the results of HSE calculations without and with SOC, respectively. (c) shows the band gap of $\mathrm{s}(\mathrm{II})-\mathrm{Au}_{2} \mathrm{Te}$ as a function of SOC strength. (d) shows the evolution of Wannier charge centers. 


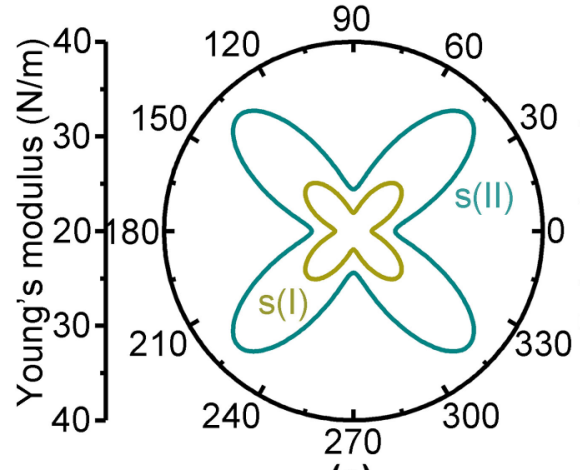

(a)

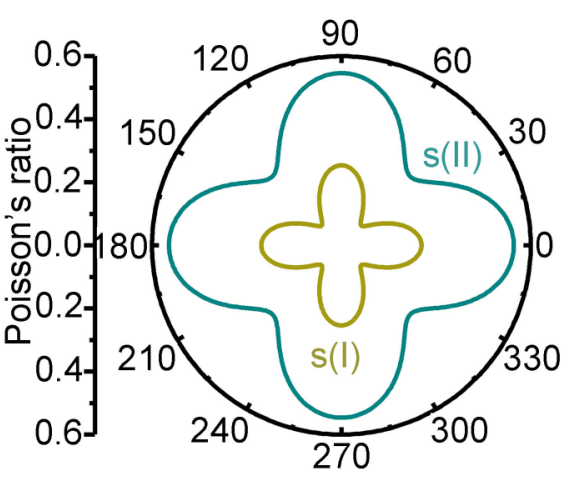

(b)

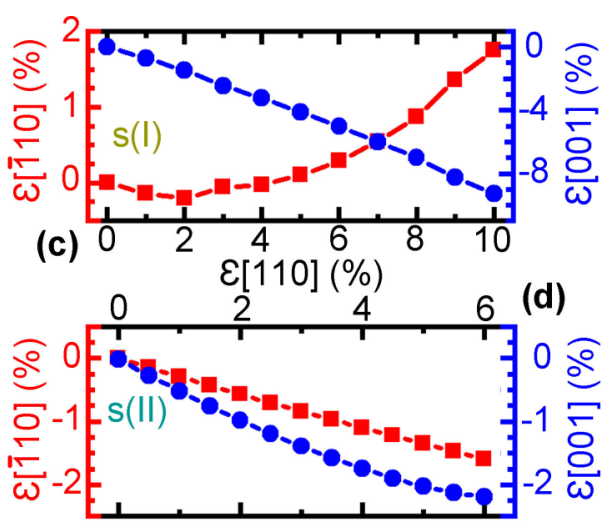

FIG. 6. (a) and (b) show the polar diagrams of in-plane Young's moduli and Poisson's ratios of s(I/II)-Au 2 Te monolayers. $\theta=0$ and $\theta=90$ correspond to the $x$ and $y$ directions, respectively. (c) and (d) show the mechanical response along the [110] direction and [001] direction under strain along the [110] direction of $2 \mathrm{D} s(\mathrm{I})-$ and $\mathrm{s}(\mathrm{II})-\mathrm{Au}_{2} \mathrm{Te}$, respectively.

Gamma point, with a direct band gap of $68.4 \mathrm{meV}$. With SOC, the spin degeneracy is lifted due to the asymmetric geometry, similarly to the case in monolayer III-Bi [73]. The lifting is contributed by the spin-orbit interaction and the bulk inversion asymmetric structure induced Rashba and Dresselhaus spinorbit terms in the effective Hamiltonian. The conduction band near the CBM splits into two bands, but the CBM is still at the $\Gamma$ point. In contrast, the highest two valence bands near the $\Gamma$ point split into four bands, and the VBM is moved away from the $\Gamma$ point. Between the CBM and VBM, there is an indirect band gap of $28.4 \mathrm{meV}$. To check whether a band inversion happens between the CBM and VBM due to the SOC effect, we perform a series of band structure calculations, in which different SOC strengths $\left(\lambda_{\mathrm{SOC}}\right)$ are employed $[13,74]$.

As shown in Fig. 5(c), with increasing SOC strengths from 0 to 0.7 , the band gap of $\mathrm{s}(\mathrm{II})-\mathrm{Au}_{2} \mathrm{Te}$ decreases. When $\lambda_{\text {SOC }}$ reaches 0.7 , a gapless electronic band structure is observed. When we continue to increase the strength of SOC, the band gap increases. The observation was also found in many other 2D materials, such as distorted hexagonal framework $\mathrm{GaBi}^{-\mathrm{Br}_{2}}(X=\mathrm{I}, \mathrm{Br}, \mathrm{Cl})$ [75], GaBi monolayer [73], and tetragonal $\mathrm{Bi}$ bilayer [13], in which the band inversion changes the $\mathbb{Z}_{2}$ and induces the topologically nontrivial nature of these materials.

To confirm the topologically nontrivial nature of s(II)$\mathrm{Au}_{2} \mathrm{Te}$, we further calculated the $\mathbb{Z}_{2}$ topological invariant. Due to the lack of inversion symmetry in $\mathrm{s}(\mathrm{II})-\mathrm{Au}_{2} \mathrm{Te}$, the parity criterion proposed by $\mathrm{Fu}$ and Kane [76] is not sufficient to get the $\mathbb{Z}_{2}$ index. A further study on the topological property was carried out within the WannierTools package [77-80]. We calculated the evolution of Wannier charge centers (WCCs) as shown in Fig. 5(d), where $\mathbb{Z}_{2}=1$ can be obtained [81]. The band inversion at the $\Gamma$ point and $\mathbb{Z}_{2}=1$ confirm that $\mathrm{s}(\mathrm{II})-\mathrm{Au}_{2} \mathrm{Te}$ is a nontrivial QSH insulator.

\section{Mechanical properties and strain-induced NPR}

The direction-dependent mechanical properties of $\mathrm{s}(\mathrm{I} / \mathrm{II})$ $\mathrm{Au}_{2} \mathrm{Te}$ can be computed from the elastic parameters we got via Eq. (2). Using the two formulas given by Cadelano et al. $[82,83]$, we can compute the in-plane Young's moduli and Poisson's ratios as

$$
E_{2 \mathrm{D}}(\alpha)=\frac{C_{11} C_{22}-C_{12}^{2}}{C_{11} \sin ^{4} \alpha+C_{22} \cos ^{4} \alpha+\left(\frac{C_{11} C_{22}-C_{12}^{2}}{C_{66}}-2 C_{12}\right) \cos ^{2} \alpha \sin ^{2} \alpha}
$$

and

$$
\vartheta(\alpha)=-\frac{\left(C_{11}+C_{22}-\frac{C_{11} C_{22}-C_{12}^{2}}{C_{66}}\right) \cos ^{2} \alpha \sin ^{2} \alpha-C_{12}\left(\cos ^{4} \alpha+\sin ^{4} \alpha\right)}{C_{11} \sin ^{4} \alpha+C_{22} \cos ^{4} \alpha+\left(\frac{C_{11} C_{22}-C_{12}^{2}}{C_{66}}-2 C_{12}\right) \cos ^{2} \alpha \sin ^{2} \alpha},
$$

respectively, in which $\alpha$ is the angle of the direction, where we define the $x$ direction as $0^{\circ}$ and the $y$ direction as $90^{\circ}$. As shown in Figs. 6(a) and 6(b), s(I/II)- $\mathrm{Au}_{2} \mathrm{Te}$ have anisotropic in-plane Young's moduli and Poisson's ratios. The softest directions of the two phases are both along the [100] and [010] directions, with their Young's moduli of $21.95 \mathrm{~N} / \mathrm{m}$ [s(I)] and $24.42 \mathrm{~N} / \mathrm{m}$ [s(II)]. The hardest directions of them are both along the [110] and [110] directions, with their Young's moduli of $26.79 \mathrm{~N} / \mathrm{m}$ [s(I)] and $37.17 \mathrm{~N} / \mathrm{m}$ [s(II)]. In com- parison, Young's modulus of graphene is as large as $335 \mathrm{~N} / \mathrm{m}$ [84]. The small in-plane Young's moduli show extraordinary flexibilities and are also observed in $\mathrm{s}-A_{2} B(A=\mathrm{Cu}, \mathrm{Ag}, \mathrm{Au}$; $B=\mathrm{S}, \mathrm{Se})$ and $\alpha-\mathrm{Ag}_{2} \mathrm{~S}$ monolayers [42,44]. The minima of the direction-dependent Poisson's ratios are both along the [110] and [110] directions whereas the maxima are along the [100] and [010] directions. In each direction, the Poisson's ratios of $\mathrm{s}(\mathrm{I})-\mathrm{Au}_{2} \mathrm{Te}$ are much smaller than that of $\mathrm{s}(\mathrm{II})$ $\mathrm{Au}_{2} \mathrm{Te}$. 


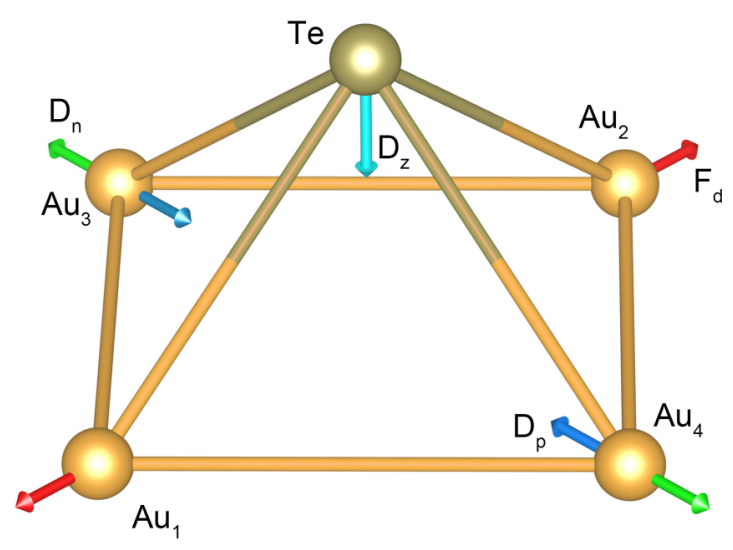

FIG. 7. The geometric changes under external tensile strain $F_{d}$ as indicated by the red vectors on atoms $\mathrm{Au}_{1}$ and $\mathrm{Au}_{2}$. The deformation of the Te atom, $D_{z}$, is indicated by the light blue vector. The deformations of atoms $\mathrm{Au}_{3}$ and $\mathrm{Au}_{4}$ in NPR and PPR cases, $D_{n}$ and $D_{p}$, are marked by the green and the blue vectors, respectively.

Auxetic materials are characterized by their unusual NPR: when a tensile/compressive strain is applied in one direction, they will expand/shrink in the vertical direction. Many attractive properties have been found in auxetic materials, such as robust shear resistance and enhanced sound absorption, which make them quite promising in the areas of medicine, clothing materials, and tough composites [85-87]. However, NPR in two-dimensional materials is rarely found [44,60,88-92]. In view of the fact that most of the other s- $A_{2} B$ structures have NPR [42], we have also computed the in-plane and out-ofplane mechanical response of $\mathrm{s}(\mathrm{I} / \mathrm{II})-\mathrm{Au}_{2} \mathrm{Te}$.

In these calculations, the strain $\epsilon[110]$ is defined as $\varepsilon[110]=a_{d}^{\prime} / a_{d}-1$, and $a_{d}^{\prime}$ is the length of the [110]direction diagonal of the unit cell. The mechanical responses in the [1 $1 \overline{0}$ ] and [001] directions are given by the change of the length of the [110]-direction diagonal and the height of the unit cells, respectively. As given previously in Fig. 2(a), the s(II) phase is kept stable when $a_{d}<8.4$; thus the mechanical response of $\mathrm{s}(\mathrm{II})-\mathrm{Au}_{2} \mathrm{Te}$ is computed in the range of $0 \leqslant$ $\varepsilon[110] \leqslant 6 \%$. As shown in Fig. 6(d), with increasing strain $\epsilon[110]$, the mechanical responses in the [110] and [001] directions, $\varepsilon[1 \overline{1} 0]$ and $\varepsilon$ [001], are always decreasing. According to the definition, the Poisson's ratio $v=-d \varepsilon_{/ /} / d \varepsilon_{\perp}$, in which $\varepsilon_{/ /}$and $\varepsilon_{\perp}$ are the relative strain along an arbitrary direction and its perpendicular direction. Thus both the in-plane and out-of-plane Poisson's ratios of $\mathrm{s}(\mathrm{II})-\mathrm{Au}_{2} \mathrm{Te}$ are positive. On the other hand, as shown in Fig. 6(c), the out-of-plane Poisson's ratios of $\mathrm{s}(\mathrm{I})-\mathrm{Au}_{2} \mathrm{Te}$ are also always positive like the s(II) phase, but the in-plane Poisson's ratios change in a different way. By applying the [110]-direction strain from 0 to $10 \%$, the relative strain $\varepsilon[1 \overline{1} 0]$ first decreases and reaches the minimum when $\varepsilon[110]=2 \%$. Intriguingly, then $\varepsilon[1 \overline{1} 0]$ increases until $\varepsilon[110]=10 \%$, showing unusual negative Poisson's ratios.

The strain-induced NPR of this type of material can be explained by the competition between the $\mathrm{Au}-\mathrm{Au}$ and $\mathrm{Au}-\mathrm{Te}$ atomic interactions [93]. We can analyze the mechanism with the help of Fig. 7. Without considering the $\mathrm{Au}-\mathrm{Te}$ atomic interaction, under the external tensile strain $F_{d}$ in the diagonal direction, the $\mathrm{Au}-\mathrm{Au}$ bond length increases, and the

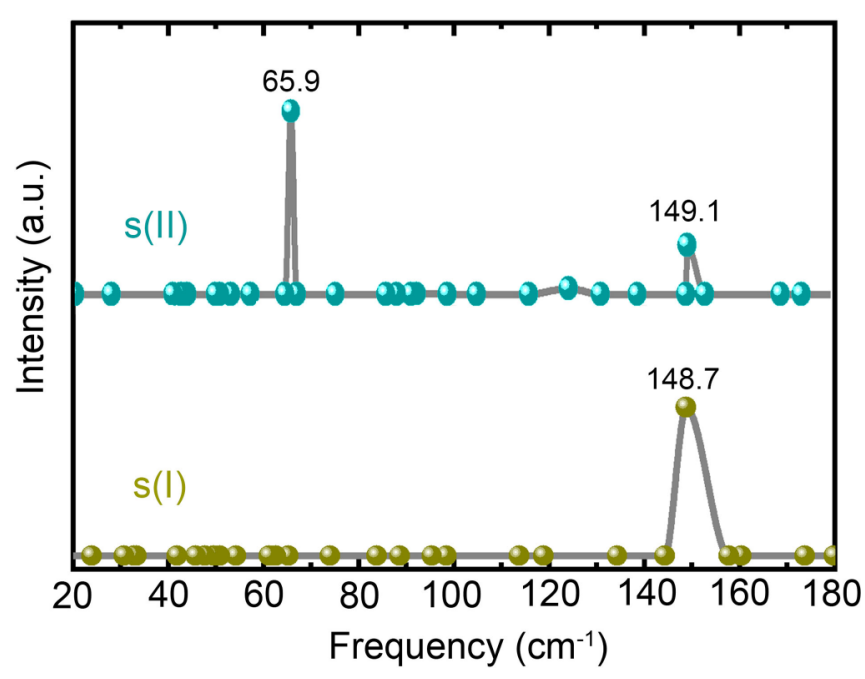

FIG. 8. The theoretically calculated Raman spectra of s(I)- and $\mathrm{s}(\mathrm{II})-\mathrm{Au}_{2} \mathrm{Te}$. The exact frequencies corresponding to the peaks are shown.

$\mathrm{Au}_{3}$ and $\mathrm{Au}_{4}$ will move in the $D_{p}$ direction, resulting in a positive Poisson's ratio (PPR). Considering the $\mathrm{Au}-\mathrm{Te}$ atomic interaction and without considering the interaction between $\mathrm{Au}-\mathrm{Au}$ atoms, with $F_{d}$, the Te atom will move downward, and the repulsive interaction will force the atoms $\mathrm{Au}_{3}$ and $\mathrm{Au}_{4}$ to move in the $D_{n}$ direction, resulting in the NPR property. Combining the two simple phenomena, for the s(I) structure with $\epsilon_{[110]}<2 \%$, the attractive interaction among Au atoms plays a dominant role, resulting in PPR. For the s(I) structure with $\epsilon_{[110]}>2 \%$, the repulsion interaction between $\mathrm{Au}$ and $\mathrm{Te}$ atoms plays a dominant role, resulting in NPR.

\section{E. Characterization with Raman spectra}

Characterization of the structures is an important part of the studies of PTMs. A good characterization method can distinguish similar phases and provide evidence of the phase transition in the experiment. Raman spectroscopy is widely used in this field [94]. In the study of $\mathrm{MoTe}_{2}$ by Wang et al. [19], the Raman spectra have clearly shown the difference between the $2 H$ phase and the $1 T^{\prime}$ phase of $\mathrm{MoTe}_{2}$, and have further shown the phase transition process. In the work of Wang et al. [95], Raman spectra have shown the phase transition process between the charge density wave phase and $1 T$ phase $\mathrm{TaS}_{2}$.

In this work, based on first-principles calculations combined with the "vasp_raman.py" code [19,96,97], we have also computed and compared the Raman spectra of the $\mathrm{s}(\mathrm{I})$ and $\mathrm{s}$ (II) phases of $\mathrm{Au}_{2} \mathrm{Te}$, as shown in Fig. 8. In the Raman spectrum of $\mathrm{s}(\mathrm{I})-\mathrm{Au}_{2} \mathrm{Te}$, there is one prominent peak at $148.7 \mathrm{~cm}^{-1}$ whereas for $\mathrm{s}(\mathrm{II})-\mathrm{Au}_{2} \mathrm{Te}$, there are two significant peaks: one is at $65.9 \mathrm{~cm}^{-1}$, and the other one is at $149.1 \mathrm{~cm}^{-1}$. The most apparent difference between these two spectra is the appearance of a peak at about $65.9 \mathrm{~cm}^{-1}$ for the $\mathrm{s}(\mathrm{I})$ phase; the corresponding phonon mode is visualized and shown in the SM (the movie file "Raman.mp4"). This mode actually corresponds to the phase transition from s(II) to s(I), which can be softened by diagonal strain, as shown in Fig. 3(b). Thus 
the Raman spectroscopy is proved to be an excellent method to distinguish the two phases of $\mathrm{Au}_{2} \mathrm{Te}$.

\section{SUMMARY}

In summary, we systematically investigated the $2 \mathrm{D}$ forms of $\mathrm{Au}_{2} \mathrm{Te}$ and predicted two stable $\mathrm{Au}_{2} \mathrm{Te}$ phases, $\mathrm{s}(\mathrm{I})$ - and $\mathrm{s}(\mathrm{II})-\mathrm{Au}_{2} \mathrm{Te}$, which are similar in both geometries and potential energies. Their energetic stability is studied and the mechanical, dynamical, and thermal stability is confirmed. Notably, the two phases have quite different electronic and mechanical properties. The $\mathrm{s}(\mathrm{I})-\mathrm{Au}_{2} \mathrm{Te}$ monolayer is a directgap semiconductor, with a gap of $1.252 \mathrm{eV}$. The carrier mobilities of the $\mathrm{s}(\mathrm{I})-\mathrm{Au}_{2} \mathrm{Te}$ monolayer are as high as $3.45 \times$ $10^{4} \mathrm{~cm}^{2} /(\mathrm{V} \mathrm{s})$ (electron) and $6.47 \times 10^{3} \mathrm{~cm}^{2} /(\mathrm{V} \mathrm{s})$ (hole), superior to black phosphorus and $1 H-\mathrm{MoS}_{2}$. In contrast, the $\mathrm{s}(\mathrm{II})-\mathrm{Au}_{2} \mathrm{Te}$ monolayer is a topological insulator with $\mathbb{Z}_{2}=1$. The nontrivial indirect band gap is $28.4 \mathrm{meV}$, which makes $\mathrm{s}(\mathrm{II})-\mathrm{Au}_{2} \mathrm{Te}$ a room-temperature topological insulator. By applying strain along the [110] direction, an unusual in-plane negative Poisson ratio can be achieved in $s(\mathrm{I})-\mathrm{Au}_{2} \mathrm{Te}$, but in $\mathrm{s}(\mathrm{II})-\mathrm{Au}_{2} \mathrm{Te}$, the Poisson ratio is always positive. Most interestingly, the structural phase transition between the semiconducting phase and the topological insulator phase can be achieved with the help of commonly used methods in experiments. By applying tensile strain, a $\mathrm{s}(\mathrm{II}) \rightarrow \mathrm{s}(\mathrm{I})$ phase transition can be achieved. On the other hand, since s(II)$\mathrm{Au}_{2} \mathrm{Te}$ has lower energy than $\mathrm{s}(\mathrm{I})$, a $\mathrm{s}(\mathrm{I}) \rightarrow \mathrm{s}(\mathrm{II})$ phase transition can be expected by chemical means. Moreover, it is found that the electric field can significantly change the energetic relationship of $s(\mathrm{I})-$ and $s(\mathrm{II})-\mathrm{Au}_{2} \mathrm{Te}$, which indicates that the $\mathrm{s}(\mathrm{I}) \rightarrow \mathrm{s}(\mathrm{II})$ phase transition can also be achieved by electrostatic gating with the assistance of heat. The results above show that $\mathrm{s}(\mathrm{I} / \mathrm{II})-\mathrm{Au}_{2} \mathrm{Te}$ monolayers are a new class of 2D materials exhibiting transformation between topological insulator and semiconducting phases, which could be further explored for use in phase-change electronic devices.

\section{ACKNOWLEDGMENTS}

We thank Yuhang Liu, Duo Wang, and Chin Shen Ong for their help and useful discussions. This work is supported by Project Grant No. 2016-05366 and Swedish Research Links Program Grant No. 2017-05447 from the Swedish Research Council. L.L. acknowledges financial support from the National Natural Science Foundation of China (Grant No. 12004097), the Natural Science Foundation of Hebei Province (Grant No. A2020202031), and the Foundation for the Introduced Overseas Scholars of Hebei Province (Grant No. C20200313). X.C. thanks the China Scholarship Council for financial support (Grant No. 201606220031). We also acknowledge the SNIC-UPPMAX, SNIC-HPC2N, and SNICNSC centers under the Swedish National Infrastructure for Computing (SNIC) resources for the allocation of time on high-performance supercomputers. Moreover, supercomputing resources from PRACE DECI-15 project DYNAMAT are gratefully acknowledged.
[1] K. S. Novoselov, A. K. Geim, S. V. Morozov, D. Jiang, Y. Zhang, S. V. Dubonos, I. V. Grigorieva, and A. A. Firsov, Science 306, 666 (2004).

[2] K. S. Novoselov, A. K. Geim, S. V. Morozov, D. Jiang, M. I. Katsnelson, I. V. Grigorieva, S. V. Dubonos, and A. A. Firsov, Nature (London) 438, 197 (2005).

[3] Y. Zhang, Y.-W. Tan, H. L. Stormer, and P. Kim, Nature (London) 438, 201 (2005).

[4] C. L. Kane and E. J. Mele, Phys. Rev. Lett. 95, 226801 (2005).

[5] X.-L. Qi and S.-C. Zhang, Rev. Mod. Phys. 83, 1057 (2011).

[6] B. A. Bernevig and S.-C. Zhang, Phys. Rev. Lett. 96, 106802 (2006).

[7] M. Z. Hasan and C. L. Kane, Rev. Mod. Phys. 82, 3045 (2010).

[8] M. König, S. Wiedmann, C. Brüne, A. Roth, H. Buhmann, L. W. Molenkamp, X.-L. Qi, and S.-C. Zhang, Science 318, 766 (2007).

[9] I. Knez, R.-R. Du, and G. Sullivan, Phys. Rev. Lett. 107, 136603 (2011).

[10] Z. Fei, T. Palomaki, S. Wu, W. Zhao, X. Cai, B. Sun, P. Nguyen, J. Finney, X. Xu, and D. H. Cobden, Nat. Phys. 13, 677 (2017).

[11] X. Qian, J. Liu, L. Fu, and J. Li, Science 346, 1344 (2014).

[12] Z.-Q. Huang, M.-L. Xu, G. Macam, C.-H. Hsu, and F.-C. Chuang, Phys. Rev. B 102, 075306 (2020).

[13] L. Kou, X. Tan, Y. Ma, H. Tahini, L. Zhou, Z. Sun, D. Aijun, C. Chen, and S. C. Smith, 2D Mater. 2, 045010 (2015).
[14] X. Zhang, Z. Wang, M. Zhao, and F. Liu, Phys. Rev. B 93, 165401 (2016).

[15] P. Chen, W. W. Pai, Y.-H. Chan, W.-L. Sun, C.-Z. Xu, D.-S. Lin, M. Y. Chou, A.-V. Fedorov, and T.-C. Chiang, Nat. Commun. 9, 2003 (2018).

[16] C. Zhang, Santosh KC, Y. Nie, C. Liang, W. G. Vandenberghe, R. C. Longo, Y. Zheng, F. Kong, S. Hong, R. M. Wallace, and K. Cho, ACS Nano 10, 7370 (2016).

[17] Q. Wang, E. T. F. Rogers, B. Gholipour, C.-M. Wang, G. Yuan, J. Teng, and N. I. Zheludev, Nat. Photonics 10, 60 (2016).

[18] M. Wuttig and N. Yamada, Nat. Mater. 6, 824 (2007).

[19] Y. Wang, J. Xiao, H. Zhu, Y. Li, Y. Alsaid, K. Y. Fong, Y. Zhou, S. Wang, W. Shi, Y. Wang, A. Zettl, E. J. Reed, and X. Zhang, Nature (London) 550, 487 (2017).

[20] N. Shukla, A. V. Thathachary, A. Agrawal, H. Paik, A. Aziz, D. G. Schlom, S. K. Gupta, R. Engel-Herbert, and S. Datta, Nat. Commun. 6, 7812 (2015).

[21] M. J. Hollander, Y. Liu, W.-J. Lu, L.-J. Li, Y.-P. Sun, J. A Robinson, and S. Datta, Nano Lett. 15, 1861 (2015).

[22] D. H. Keum, S. Cho, J. H. Kim, D.-H. Choe, H.-J. Sung, M. Kan, H. Kang, J.-Y. Hwang, S. W. Kim, H. Yang, K. J. Chang, and Y. H. Lee, Nat. Phys. 11, 482 (2015).

[23] M. Vellinga, R. de Jonge, and C. Haas, J. Solid State Chem. 2 , 299 (1970).

[24] Y. Li, K.-A. N. Duerloo, K. Wauson, and E. J. Reed, Nat. Commun. 7, 10671 (2016). 
[25] C. Kim, S. Issarapanacheewin, I. Moon, K. Y. Lee, C. Ra, S. Lee, Z. Yang, and W. J. Yoo, Adv. Electron. Mater. 6, 1900964 (2020).

[26] B. Peng, H. Zhang, W. Chen, B. Hou, Z.-J. Qiu, H. Shao, H. Zhu, B. Monserrat, D. Fu, H. Weng, and C. M. Soukoulis, npj 2D Mater. Appl. 4, 14 (2020).

[27] E. Sajadi, T. Palomaki, Z. Fei, W. Zhao, P. Bement, C. Olsen, S. Luescher, X. Xu, J. A. Folk, and D. H. Cobden, Science 362, 922 (2018).

[28] V. Fatemi, S. Wu, Y. Cao, L. Bretheau, Q. D. Gibson, K. Watanabe, T. Taniguchi, R. J. Cava, and P. Jarillo-Herrero, Science 362, 926 (2018).

[29] J. Zhou, S. Zhang, and J. Li, NPG Asia Mater. 12, 2 (2020).

[30] S. Zhang, M. Xie, B. Cai, H. Zhang, Y. Ma, Z. Chen, Z. Zhu, Z. Hu, and H. Zeng, Phys. Rev. B 93, 245303 (2016).

[31] Y. Xiao, M. Zhou, J. Liu, J. Xu, and L. Fu, Sci. China Mater. 62, 759 (2019).

[32] C. Eames and M. S. Islam, J. Am. Chem. Soc. 136, 16270 (2014).

[33] Y. Ma, A. Kuc, and T. Heine, J. Am. Chem. Soc. 139, 11694 (2017).

[34] F. B. Romdhane, O. Cretu, L. Debbichi, O. Eriksson, S. Lebègue, and F. Banhart, Small 11, 1253 (2015).

[35] B. Li, L. Huang, G. Zhao, Z. Wei, H. Dong, W. Hu, L.-W. Wang, and J. Li, Adv. Mater. 28, 8271 (2016).

[36] J. Feng, X. Li, Z. Shi, C. Zheng, X. Li, D. Leng, Y. Wang, J. Liu, and L. Zhu, Adv. Opt. Mater. 8, 1901762 (2019).

[37] Y. Zhu, L. Peng, W. Zhu, D. Akinwande, and G. Yu, Chem. Mater. 28, 4307 (2016).

[38] K. Qian, L. Gao, H. Li, S. Zhang, J. H. Yan, C. Liu, J. O. Wang, T. Qian, H. Ding, Y. Y. Zhang, X. Lin, S. X. Du, and H.-J. Gao, Chin. Phys. B 29, 018104 (2019).

[39] Y. Wang, J. Lv, L. Zhu, and Y. Ma, Phys. Rev. B 82, 094116 (2010).

[40] Y. Wang, M. Miao, J. Lv, L. Zhu, K. Yin, H. Liu, and Y. Ma, J. Chem. Phys. 137, 224108 (2012).

[41] Y. Guo, Q. Wu, Y. Li, N. Lu, K. Mao, Y. Bai, J. Zhao, J. Wang, and X. C. Zeng, Nanoscale Horiz. 4, 223 (2019).

[42] X. Chen, D. Wang, X. Liu, L. Li, and B. Sanyal, J. Phys. Chem. Lett. 11, 2925 (2020).

[43] Q. Wu, W. W. Xu, D. Lin, J. Wang, and X. C. Zeng, J. Phys. Chem. Lett. 10, 3773 (2019).

[44] R. Peng, Y. Ma, Z. He, B. Huang, L. Kou, and Y. Dai, Nano Lett. 19, 1227 (2019).

[45] A. O. Lyakhov, A. R. Oganov, H. T. Stokes, and Q. Zhu, Comput. Phys. Commun. 184, 1172 (2013).

[46] A. R. Oganov and C. W. Glass, J. Chem. Phys. 124, 244704 (2006).

[47] C. W. Glass, A. R. Oganov, and N. Hansen, Comput. Phys. Commun. 175, 713 (2006).

[48] Q. Zhu, L. Li, A. R. Oganov, and P. B. Allen, Phys. Rev. B 87, 195317 (2013).

[49] A. R. Oganov, A. O. Lyakhov, and M. Valle, Acc. Chem. Res. 44, 227 (2011).

[50] G. Kresse, J. Non-Cryst. Solids 192-193, 222 (1995).

[51] G. Kresse and J. Furthmüller, Phys. Rev. B 54, 11169 (1996).

[52] J. P. Perdew, K. Burke, and M. Ernzerhof, Phys. Rev. Lett. 77, 3865 (1996).

[53] A. Togo and I. Tanaka, Scr. Mater. 108, 1 (2015).
[54] A. V. Krukau, O. A. Vydrov, A. F. Izmaylov, and G. E. Scuseria, J. Chem. Phys. 125, 224106 (2006).

[55] V. Wang, N. Xu, J. C. Liu, G. Tang, and W.-T. Geng, VASPKIT: A user-friendly interface facilitating high-throughput computing and analysis using VASP code, arXiv:1908.08269.

[56] K. Momma and F. Izumi, J. Appl. Crystallogr. 41, 653 (2008).

[57] See Supplemental Material at http://link.aps.org/supplemental/ 10.1103/PhysRevB.103.075429 for the following parts: the enthalpies of the allotropes found in the evolutionary structure search; the simulated structural evolution process between s(II)$\mathrm{Au}_{2} \mathrm{Te}$ and $\mathrm{s}(\mathrm{I})-\mathrm{Au}_{2} \mathrm{Te}$; the LDOS of s- $\mathrm{Au}_{2} \mathrm{Te}$ monolayers; the enlarged band structure of $\mathrm{s}(\mathrm{II})-\mathrm{Au}_{2} \mathrm{Te}$; the information of the atomic position in $\mathrm{s}-\mathrm{Au}_{2} \mathrm{Te}$; the convex hull diagram of the 2D Au-Te system; the phonon spectra of s(II)- $\mathrm{Au}_{2} \mathrm{Te}$ with strain $6 \%$ and $7 \%$; the results of the isobaric-isothermic BOMD simulation of $\mathrm{s}(\mathrm{II})-\mathrm{Au}_{2} \mathrm{Te}$ with variable lattice parameters; the eigenvector of the softened phonon in $\mathrm{s}(\mathrm{II})-\mathrm{Au}_{2} \mathrm{Te}$ with strain of $6.5 \%$; the eigenvector of the phonon mode corresponding to the Raman spectra peak at $65.9 \mathrm{~cm}^{-1}$.

[58] L.-M. Yang, V. Bačić, I. A. Popov, A. I. Boldyrev, T. Heine, T. Frauenheim, and E. Ganz, J. Am. Chem. Soc. 137, 2757 (2015).

[59] R. C. Andrew, R. E. Mapasha, A. M. Ukpong, and N. Chetty, Phys. Rev. B 85, 125428 (2012).

[60] S. Zhang, J. Zhou, Q. Wang, X. Chen, Y. Kawazoe, and P. Jena, Proc. Natl. Acad. Sci. USA 112, 2372 (2015).

[61] X. Wang, Y. Gong, G. Shi, W. L. Chow, K. Keyshar, G. Ye, R. Vajtai, J. Lou, Z. Liu, E. Ringe, B. K. Tay, and P. M. Ajayan, ACS Nano 8, 5125 (2014).

[62] C. H. Naylor, W. M. Parkin, Z. Gao, H. Kang, M. Noyan, R. B. Wexler, L. Z. Tan, Y. Kim, C. E. Kehayias, F. Streller, Y. R. Zhou, R. Carpick, Z. Luo, Y. W. Park, A. M. Rappe, M. Drndić, J. M. Kikkawa, and A. T. C. Johnson, 2D Mater. 4, 021008 (2017).

[63] M. Zhao, X. Zhang, and L. Li, Sci. Rep. 5, 16108 (2015).

[64] M. Zhao, X. Chen, L. Li, and X. Zhang, Sci. Rep. 5, 8441 (2015).

[65] G.-R. Qian, X. Dong, X.-F. Zhou, Y. Tian, A. R. Oganov, and H.-T. Wang, Comput. Phys. Commun. 184, 2111 (2013).

[66] M. Parrinello and A. Rahman, Phys. Rev. Lett. 45, 1196 (1980).

[67] M. Parrinello and A. Rahman, J. Appl. Phys. 52, 7182 (1981).

[68] J. Bardeen and W. Shockley, Phys. Rev. 80, 72 (1950).

[69] S. Bruzzone and G. Fiori, Appl. Phys. Lett. 99, 222108 (2011).

[70] J. Qiao, X. Kong, Z.-X. Hu, F. Yang, and W. Ji, Nat. Commun. 5, 4475 (2014).

[71] R. Fivaz and E. Mooser, Phys. Rev. 163, 743 (1967).

[72] B. Radisavljevic, A. Radenovic, J. Brivio, V. Giacometti, and A. Kis, Nat. Nanotechnol. 6, 147 (2011).

[73] F.-C. Chuang, L.-Z. Yao, Z.-Q. Huang, Y.-T. Liu, C.-H. Hsu, T. Das, H. Lin, and A. Bansil, Nano Lett. 14, 2505 (2014).

[74] B. Huang, K.-H. Jin, H. L. Zhuang, L. Zhang, and F. Liu, Phys. Rev. B 93, 115117 (2016).

[75] L. Li, O. Leenaerts, X. Kong, X. Chen, M. Zhao, and F. M. Peeters, Nano Res. 10, 2168 (2017).

[76] L. Fu and C. L. Kane, Phys. Rev. B 76, 045302 (2007).

[77] A. A. Mostofi, J. R. Yates, G. Pizzi, Y.-S. Lee, I. Souza, D. Vanderbilt, and N. Marzari, Comput. Phys. Commun. 185, 2309 (2014). 
[78] Q. Wu, S. Zhang, H.-F. Song, M. Troyer, and A. A. Soluyanov, Comput. Phys. Commun. 224, 405 (2018).

[79] A. A. Soluyanov and D. Vanderbilt, Phys. Rev. B 83, 235401 (2011).

[80] R. Yu, X. L. Qi, A. Bernevig, Z. Fang, and X. Dai, Phys. Rev. B 84, 075119 (2011).

[81] A. A. Soluyanov and D. Vanderbilt, Phys. Rev. B 83, 035108 (2011).

[82] E. Cadelano and L. Colombo, Phys. Rev. B 85, 245434 (2012).

[83] E. Cadelano, P. L. Palla, S. Giordano, and L. Colombo, Phys. Rev. B 82, 235414 (2010).

[84] C. Lee, X. Wei, J. W. Kysar, and J. Hone, Science 321, 385 (2008).

[85] H. Kimizuka, H. Kaburaki, and Y. Kogure, Phys. Rev. Lett. 84, 5548 (2000).

[86] Y. J. Park and J. K. Kim, Adv. Mater. Sci. Eng. 2013, 853289 (2013).

[87] K. E. Evans and A. Alderson, Adv. Mater. 12, 617 (2000).

[88] Z. Gao, X. Dong, N. Li, and J. Ren, Nano Lett. 17, 772 (2017).
[89] X. Liu, X. Shao, B. Yang, and M. Zhao, Nanoscale 10, 2108 (2018).

[90] L. Kou, Y. Ma, C. Tang, Z. Sun, A. Du, and C. Chen, Nano Lett. 16, 7910 (2016).

[91] J.-W. Jiang and H. S. Park, Nat. Commun. 5, 4727 (2014).

[92] C. Zhang, T. He, S. K. Matta, T. Liao, L. Kou, Z. Chen, and A. Du, J. Phys. Chem. Lett. 10, 2567 (2019).

[93] J. Pan, Y.-F. Zhang, J. Zhang, H. Banjade, J. Yu, L. Yu, S. Du, A. Ruzsinszky, Z. Hu, and Q. Yan, npj Comput. Mater. 6, 154 (2020).

[94] X. Wen, Y. Liu, D. Xu, Y. Zhao, R. K. Lake, and J. Guo, J. Phys. Chem. Lett. 11, 1589 (2020).

[95] X. Wang, H. Liu, J. Wu, J. Lin, W. He, H. Wang, X. Shi, K. Suenaga, and L. Xie, Adv. Mater. 30, 1800074 (2018).

[96] A. Fonari and S. Stauffer, vasp_raman.py, https://github.com/ raman-sc/VASP.

[97] A. Fonari, N. S. Corbin, D. Vermeulen, K. P. Goetz, O. D. Jurchescu, L. E. McNeil, J. L. Bredas, and V. Coropceanu, J. Chem. Phys. 143, 224503 (2015). 